\title{
HYPERTROPHIC OBSTRUCTIVE CARDIOMYOPATHY IN PEDIATRIC PATIENTS: RESULTS OF SURGICAL TREATMENT
}

David A. Theodoro, $\mathrm{MD}^{\mathrm{a}}$

Gordon K. Danielson, $\mathrm{MD}^{\mathrm{a}}$

Robert H. Feldt, MD ${ }^{\mathrm{b}}$

Betty J. Anderson, RN ${ }^{\mathrm{a}}$
Between April 1975 and May 1995, 25 pediatric patients on one hospital service underwent extended left ventricular septal myectomy because of hypertrophic obstructive cardiomyopathy. Ages ranged from 2 months to 20 years (mean, 11.2 years). Seventeen patients had moderate to severe mitral valve insufficiency. Medical therapy had failed in all patients and one patient had undergone dual-chamber pacemaker implantation without improvement. Left ventricular outflow tract gradients ranged from 50 to $154 \mathrm{~mm} \mathrm{Hg}$ (mean, $99.9 \pm 25.2$ ). Concomitant cardiac procedures included mitral valve repair $(n=2)$, automatic implantable cardioverter defibrillator implantation $(n=1)$, and closure of atrial septal defect $(n=1)$. Intraoperative premyectomy left ventricular outflow tract gradients ranged from 20 to $117 \mathrm{~mm} \mathrm{Hg}$ (mean, $60.4 \pm 26.2$ ) and postmyectomy gradients ranged from 0 to $20 \mathrm{~mm} \mathrm{Hg}$ (mean, $6.6 \pm 5.9$ ). Postmyectomy mitral insufficiency was reduced to a regurgitant fraction of $0 \%$ to $12 \%$, and no patient required mitral valve replacement. One patient required a pacemaker because of complete heart block; on subsequent follow-up, normal sinus rhythm had returned. There was no early mortality and no instance of aortic or mitral valve injury or ventricular septal defect. Follow-up ranged from 10 months to 20 years (mean, 6.4 years). There were no late deaths. Left ventricular outflow tract gradients by echocardiography were a mean of $14.2 \mathrm{~mm} \mathrm{Hg}$ with a median of $5.0 \mathrm{~mm} \mathrm{Hg}$. All patients had normal sinus rhythm. Reoperation because of recurrent left ventricular outflow tract obstruction was necessary in two patients at 3.2 years and 12.4 years after initial myectomy, respectively. All patients but one have New York Heart Association class I or II function. We conclude that extended septal myectomy is a safe and effective means of relieving cardiac symptoms and left ventricular outflow tract obstruction in pediatric patients with severe hypertrophic obstructive cardiomyopathy unresponsive to medical management, and late survivorship compares favorably with the natural history of the disease. (J Thorac Cardiovasc Surg 1996;112:1589-99)
$\mathrm{H}$ ypertrophic obstructive cardiomyopathy (HOCM) is an intriguing primary myocardial disease based on distinct genetic mutations. ${ }^{1,2}$ The heterogeneity of

From the Division of Thoracic and Cardiovascular Surgery ${ }^{\mathrm{a}}$ and the Section of Pediatric Cardiology, ${ }^{b}$ Mayo Clinic/Foundation, Rochester, Minn.

Read at the Seventy-sixth Annual Meeting of The American Association for Thoracic Surgery, San Diego, Calif., April 28-May 1, 1996.

Received for publication May 6, 1996; revisions requested June 10, 1996; revisions received July 12, 1996; accepted for publication July 15, 1996.

Address for reprints: Gordon K. Danielson, MD, Mayo Clinic, 200 First St. SW, Rochester, MN 55905.

Copyright $(\mathcal{C} 1996$ by Mosby-Year Book, Inc.

$0022-5223 / 96 \$ 5.00+0 \quad \mathbf{1 2 / 6 / 7 6 5 1 1}$ this disease process is demonstrated by its wide variation in clinical symptoms, hemodynamic characteristics, natural history, age at presentation, and risk for sudden death. For patients at our institution who have severe HOCM that is unresponsive to medical management, an extended left ventricular septal myectomy (LVM) has been offered. ${ }^{3}$ Several recent surgical series have confirmed the effectiveness of LVM in reducing symptoms and systolic pressure gradients across the left ventricular outflow tract (LVOT), and some of the data indicate an improvement in late patient survival. ${ }^{4-8}$ Most surgical reports include only adults or patients over a wide age spectrum without separate analysis of results for the pediatric subset. This current study assesses the results in a series of pediatric patients who underwent LVM because of 


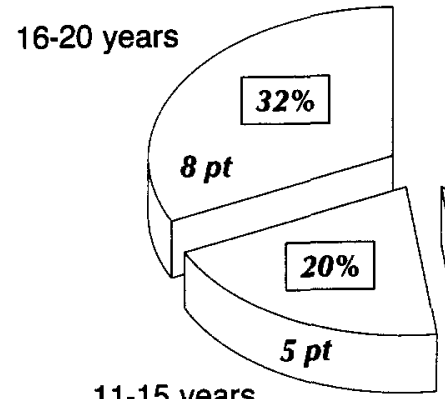

$11-15$ years

Fig. 1. Pie chart of age distribution. pt, Patients.

severe HOCM on one surgical service during a 20 -year interval.

\section{Patients and methods}

Patients. Between April 1975 and May 1995, 25 patients (13 male and 12 female) who were 20 years old or younger underwent primary operation for HOCM on one surgical service. Ages ranged from 2 days to 19 years (mean, 7.7 years; median, 9.0 years) at diagnosis and from 2 months to 20 years (mean, 11.2 years; median, 14.0 years) at operation (Fig. 1). The diagnosis of HOCM was based on clinical evaluation and on echocardiography or cardiac catheterization, or both. Patients with severe obstruction of both right and left ventricular outflow tracts were excluded and their cases will be reported separately. Preoperative clinical and hemodynamic data are summarized in Table I. In 20 patients (80\%), the indication for operation was refractory symptoms despite maximal medical therapy (Table I); duration of treatment ranged from 2.6 months to 7.2 years (mean, 2.2 years). Five patients were free of symptoms; these patients had resting LVOT gradients of $80 \mathrm{~mm} \mathrm{Hg}$ or more and were thought to be at increased risk for death, similar to patients with severe valvular aortic stenosis. All patients had preoperative resting or inducible LVOT gradients of $50 \mathrm{~mm} \mathrm{Hg}$ or more. One patient had undergone dual-chamber pacemaker implantation elsewhere without improvement of symptoms or LVOT gradient.

Associated cardiac diagnoses included moderate to severe mitral valve insufficiency $(n=17)$, mild aortic insufficiency $(n=3)$, atrial septal defect $(n=1)$, bicuspid aortic valve $(n=1)$, and muscle bridge over the left anterior descending coronary artery $(n=1)$. Three patients had a history of serious ventricular arrhythmia (ventricular tachycardia, $n=2$; easily inducible ventricular fibrillation on electrophysiologic study, $n=1$ ). Echocardiographic gradients were measured as maximum instantaneous gradients and cardiac catheterization gradients were measured as peak systolic gradients. Preoperative gradients ranged from 50 to $154 \mathrm{~mm} \mathrm{Hg}$ (mean, $99.9 \pm 25.2)$ as determined by echocardiography $(n=12)$, cardiac catheterization $(n=4)$, or both $(n=9)$.

Current surgical techniques. Pressures are measured in all four cardiac chambers and in the pulmonary artery and aorta before the start of cardiopulmonary bypass. Standard cardiopulmonary bypass with moderate $\left(25^{\circ}\right.$ to $32^{\circ} \mathrm{C}$ ) hypothermia is used. Protecting the severely hypertrophied myocardium during aortic crossclamping is especially important. We prefer multiple-dose cold blood cardioplegia combined with topical cooling. A transverse aortotomy is made anteriorly and the incision is extended rightward into the noncoronary sinus. The aortic valve cusps are retracted to expose the hypertrophied septum and region of septal contact with the anterior leaflet of the mitral valve, which typically produces fibrotic endocardial thickening (friction lesion). The thickness and extent of the septal hypertrophy are evaluated by intraoperative transesophageal echocardiography, supplemented as indicated with bimanual palpation of the heart between the LVOT and the anterior surface of the left ventricle.

The septal resection is begun by making two parallel longitudinal incisions in the septum, the first beneath the nadir of the right coronary cusp and the second beneath the commissure between the right and left cusps (Fig. 2). Accurate placement of these incisions protects the main conduction bundle. The incisions are connected superiorly with a third incision that is continued into the septum, and a generous wedge of muscle is resected. The resection is continued apically to at least the level of the papillary muscles. Exposure of the septum may be facilitated by posterior displacement of the anterior wall of the left ventricle. The myocardial groove in the septum is deepened as necessary by further resection with a scalpel or pituitary rongeur; if exposure is difficult, the groove in the septum is incised with a scalpel and widened by digital manipulation. Additional myocardium is resected from this groove leftward to the anterior leaflet of the mitral valve. For patients who have associated midventricular obstruction, further muscle resection is done around and below the papillary muscles toward the apex. These surgical techniques collectively are termed an extended septal myectomy. The adequacy and distal extent of the resection are evaluated by inspection and digital palpation. Occasionally, a distorted anterior mitral leaflet is repaired by a plicating suture, ${ }^{9}$ and an anterior papillary muscle that appears to be located too far anteriorly is released by an incision made anterior to the base of the muscle. ${ }^{10,11}$

Placement of an insulating pad behind the left ventricle permits safe rewarming during aortic closure. We prefer to defibrillate the heart with warm blood cardioplegic solution rather than electric defibrillation, which sometimes requires multiple shocks. Pressure measurements are repeated in the left ventricle and ascending aorta after the patient is weaned from cardiopulmonary bypass and hemodynamic conditions are stabilized. The degree of mitral valve insufficiency is assessed before and after cardiopulmonary bypass by transesophageal echocardiography, often supplemented with double-sampling dye curves done by injection of indocyanine green dye into the apex of the left ventricle and sampling simultaneously in the dome of the left atrium and the aorta, a technique that provides more accurate quantitation of the mitral insufficiency.

Statistical analysis. Follow-up information was obtained from correspondence with patients and referring physicians and from telephone interviews. Postoperative freedom from reoperation was calculated by the Kaplan-Meier method. 
Table I. Clinical and hemodynamic data

\begin{tabular}{|c|c|c|c|c|c|}
\hline & \multicolumn{2}{|c|}{ Before myectomy } & \multicolumn{2}{|c|}{ After myectomy } & \multirow[b]{2}{*}{$p$ Value } \\
\hline & No. & $\%$ & No. & $\%$ & \\
\hline \multicolumn{6}{|l|}{ NYHA class } \\
\hline I & 5 & 20 & 21 & 84 & \\
\hline II & 10 & 40 & 3 & 12 & \\
\hline III & 9 & 36 & 1 & 4 & \\
\hline IV & 1 & 4 & 0 & 0 & \\
\hline Mean & \multicolumn{2}{|c|}{$2.24 \pm 0.83$} & \multicolumn{2}{|c|}{$1.12 \pm 0.44$} & $<0.01$ \\
\hline \multicolumn{6}{|l|}{ Symptoms } \\
\hline Dyspnea & 18 & 72 & 4 & 16 & $<0.001$ \\
\hline Angina & 12 & 48 & 0 & 0 & $<0.001$ \\
\hline Syncope & 5 & 20 & 0 & 0 & \\
\hline Palpitations & 2 & 8 & 0 & 0 & \\
\hline \multicolumn{6}{|l|}{ Hemodynamics } \\
\hline Mean LVOT gradient $(\mathrm{mm} \mathrm{Hg})$ & \multirow{2}{*}{\multicolumn{2}{|c|}{$\begin{array}{l}99.9 \pm 25.2 \\
60.4 \pm 26.2\end{array}$}} & \multirow{2}{*}{\multicolumn{2}{|c|}{$\begin{aligned} 14.2 & \pm 27.7 \\
6.6 & \pm 5.9\end{aligned}$}} & $<0.0001$ \\
\hline Mean intraoperative LVOT gradient $(\mathrm{mm} \mathrm{Hg})$ & & & & & $<0.0001$ \\
\hline Moderate or severe MV insufficiency & 17 & 68 & 1 & 4 & $<0.0001$ \\
\hline \multicolumn{6}{|l|}{ Rhythm } \\
\hline Normal sinus & 25 & 100 & 25 & 100 & \\
\hline \multicolumn{6}{|l|}{ Medications } \\
\hline$\beta$-Adrenergic antagonists & 17 & 68 & 16 & 64 & \\
\hline Calcium channel antagonists & 4 & 16 & 6 & 24 & \\
\hline Both of the above & 3 & 12 & 2 & 8 & \\
\hline Disopyramide & 1 & 5 & 0 & 0 & \\
\hline
\end{tabular}

$M V$, Mitral valve.

The rank sum test was used to compare continuous variables. Statistical significance was accepted at $p<0.05$.

\section{Results}

Early results. A primary transaortic extended LVM was done in all 25 patients. Concomitant cardiac procedures included plication of the anterior leaflet of the mitral valve $(n=2)$, automatic implantable cardioverter defibrillator (AICD) implantation $(n=1)$, closure of atrial septal defect $(n=1)$, and unroofing of a coronary artery muscle bridge $(n=1)$. Aortic crossclamp times ranged from 11 to 53 minutes (mean, 35.3). Intraoperative LVOT gradients ranged from 20 to $117 \mathrm{~mm} \mathrm{Hg}$ (mean, $60.4 \pm 26.2$ ) before myectomy and from 0 to $20 \mathrm{~mm}$ $\mathrm{Hg}$ (mean, $6.6 \pm 5.9)$ after myectomy $(p<0.0001)$. No patients in this group required reinstitution of cardiopulmonary bypass for relief of significant residual LVOT gradient after initial LVM; 22 patients $(88 \%)$ had a gradient of $15 \mathrm{~mm} \mathrm{Hg}$ or less.

The moderate or severe mitral regurgitation present before operation in 17 patients with HOCM (Table I) was successfully eliminated in 16 patients with LVM alone $(n=14)$ or LVM combined with an anterior mitral leaflet plication $(n=2)$. None of the 16 patients showed significant (greater than
$20 \%$ ) mitral insufficiency by double-sampling dye curves. Moderate residual mitral insufficiency was present in the 2-month-old infant. No patients required mitral valve replacement.

There were no early deaths. Two patients required temporary intraaortic balloon pump support to wean them from cardiopulmonary bypass. Two patients required placement of an AICD after episodes of ventricular tachycardia or fibrillation at 1 and 2 weeks after operation, respectively; one of the two patients was known to have spontaneous ventricular tachycardia before operation. One patient underwent placement of a pacemaker because of complete heart block that was still present on postoperative day 8; on subsequent follow-up 1 year later he had normal sinus rhythm. Acute constrictive pericarditis developed in one patient who had received AICD patches intraoperatively and this necessitated pericardiectomy on postoperative day 8 . There were no instances of aortic or mitral valve injury or surgically created ventricular septal defect.

Hospital stay ranged from 5 to 48 days (median, 8; mean, 13.1). For those patients who did not require subsequent pericardiectomy, pacemaker, or AICD, the mean hospital stay was 8.5 days. Twenty patients were discharged from the hospital receiving $\beta$-ad- 


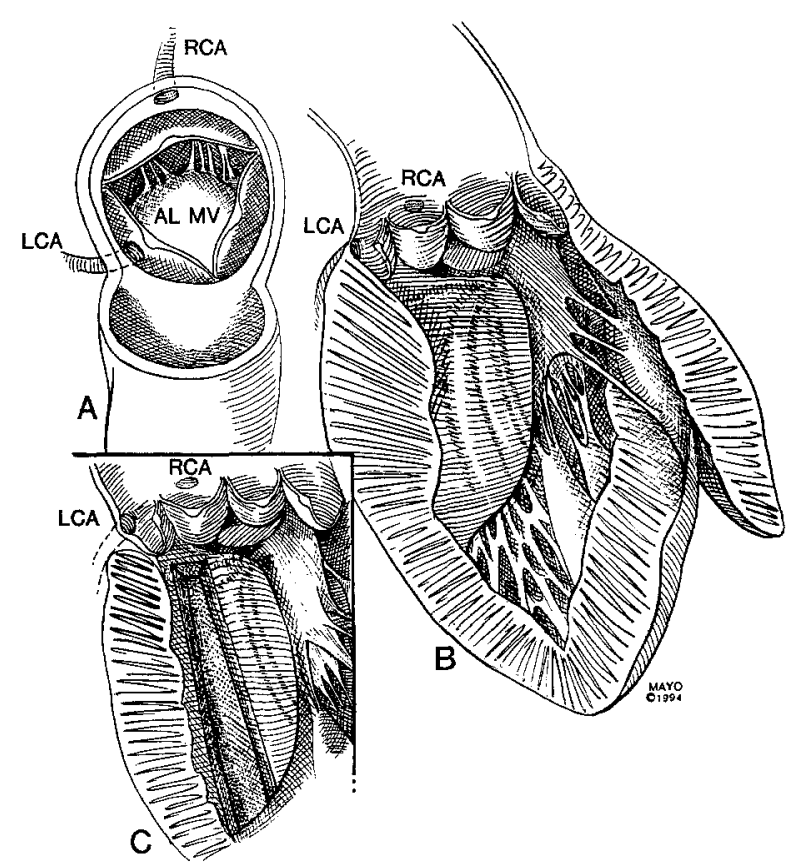

Fig. 2. Anatomic characteristics of hypertrophic obstructive cardiomyopathy. A, Surgeon's view through transverse aortotomy. Right coronary cusp is located anteriorly and left cusp is located posteriorly and to left. Gentle retraction of aortic cusps, combined with posterior displacement of left ventricle as needed, exposes ventricular septum and anterior leaflet of mitral valve $(A L M V)$. B, Sagittal view through opened left ventricle showing membranous septum below commissure between right and noncoronary cusps, distribution of left bundle branches, septal hypertrophy, and anterior leaflet of mitral valve with its subvalvular apparatus. C, Extent of initial septal resection is shown. Resection is then extended leftward to anterior leaflet of mitral valve and apically to relieve all midventricular obstruction. $R C A$, Right coronary artery; $L C A$, left coronary artery.

renergic antagonists, four receiving calcium channel antagonists, and one receiving digoxin and amiodarone.

Late results. Follow-up was obtained in all 25 patients and ranged from 10 months to 20 years (mean, $6.4 \pm 5.2$ years; median, 4.4 years) for a total of 159.5 patient-years. Ages at the time of most recent contact ranged from 3.9 to 40.9 years (mean, 17.5). There were no late deaths.

Recurrent symptoms and hemodynamically significant LVOT gradients developed in two patients necessitating reoperation at 3.2 and 12.4 years after initial myectomy, respectively. Freedom from reoperation because of recurrent LVOT obstruction was $94.4 \% \pm 5.4 \%$ at 5 years (Fig. 3). The first patient

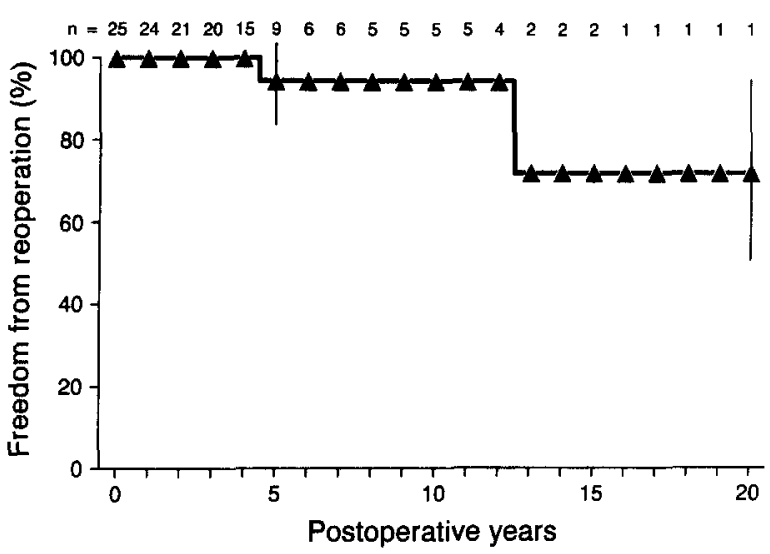

Fig. 3. Diagram of freedom from reoperation because of recurrent LVOT obstruction.

who required reoperation was 1 year old when HOCM was diagnosed. At age 2 years, echocardiography and cardiac catheterization showed a resting LVOT gradient of $100 \mathrm{~mm} \mathrm{Hg}$, and the patient underwent LVM. The intraoperative postresection LVOT gradient was $7 \mathrm{~mm} \mathrm{Hg}$, and there was no detectable mitral valve insufficiency. The patient did well during the following 2.5 years until exertional dyspnea and mild chest pain developed. Cardiac catheterization and echocardiography demonstrated an outflow gradient of $80 \mathrm{~mm} \mathrm{Hg}$. At age 5 years, 3.2 years after initial LVM, she underwent repeat myectomy. Seven years later she is completely free of symptoms and has an LVOT gradient of only $12 \mathrm{~mm}$ $\mathrm{Hg}$.

The second patient who required reoperation was diagnosed with HOCM in infancy. At age $3 \frac{1}{2}$ years she had significant symptoms (New York Heart Association [NYHA] class III) with an LVOT gradient of $60 \mathrm{~mm} \mathrm{Hg}$. LVM was done; the intraoperative postresection LVOT gradient was $15 \mathrm{~mm} \mathrm{Hg}$ and there was no evidence of important mitral or aortic valve insufficiency. She did well during the following 10 years until routine follow-up echocardiography demonstrated increasing LVOT obstruction ( $50 \mathrm{~mm} \mathrm{Hg}$ gradient). During the next 2 years, the LVOT gradient increased to $80 \mathrm{~mm} \mathrm{Hg}$ and significant dyspnea and chest pain developed. She also had severe, unexplained aortic insufficiency. At age 16 years, 12.4 years after initial LVM, she underwent reoperation for myectomy, aortoventriculoplasty (Konno procedure), and aortic valve replacement with an aortic homograft. Intraoperatively, there was massive septal hypertrophy and the previous resection site had completely filled in with 


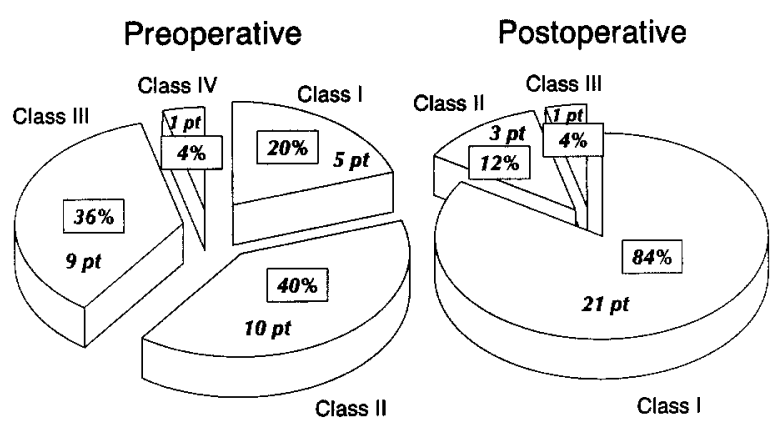

Fig. 4. Pie charts showing preoperative NYHA functional classification (left panel) and NYHA classification at most recent contact (right panel). pt, Patients.

septal tissue. She is currently a 20 -year-old college student and is completely free of symptoms with an LVOT gradient of $12 \mathrm{~mm} \mathrm{Hg}$.

A third patient was born with severe cardiac failure; the diagnosis of HOCM was made on the second day of life (she was in NYHA class IV). She was initially treated medically, but because of persistent cardiac failure, severe mitral valve insufficiency, and an LVOT gradient of $61 \mathrm{~mm} \mathrm{Hg}$, she underwent LVM at age 2 months. The postresection intraoperative LVOT gradient was 16 $\mathrm{mm} \mathrm{Hg}$; there was no gradient across the right ventricular outflow tract. Mitral insufficiency was graded as moderate. At the time of hospital discharge, echocardiography detected an LVOT gradient of $40 \mathrm{~mm} \mathrm{Hg}$ and moderate mitral valve insufficiency. She was treated with $\beta$-adrenergic antagonists and calcium channel antagonists, but during the next 5 years severe LVOT and right ventricular outflow tract gradients developed (130 and $41 \mathrm{~mm} \mathrm{Hg}$, respectively) and she was in functional class III. Because of the progressive global nature of the HOCM in this case, the resulting severe biventricular outflow tract obstructions, and the high risk associated with biventricular septal resection in pediatric patients, this patient recently underwent successful heart transplantation.

Of the remaining 24 patients, 21 were free of symptoms (NYHA class I) at the time of most recent contact, and 3 had only mild limitations (NYHA class II) (Fig. 4). The mean NYHA functional class was $2.24 \pm 0.83$ before operation and $1.12 \pm 0.44$ after operation $(p<0.01$ by the paired $t$ test). The prevalence of angina, syncope, and dyspnea decreased substantially: $14(78 \%)$ of 18 patients with dyspnea $(p<0.001), 12(100 \%)$ of 12

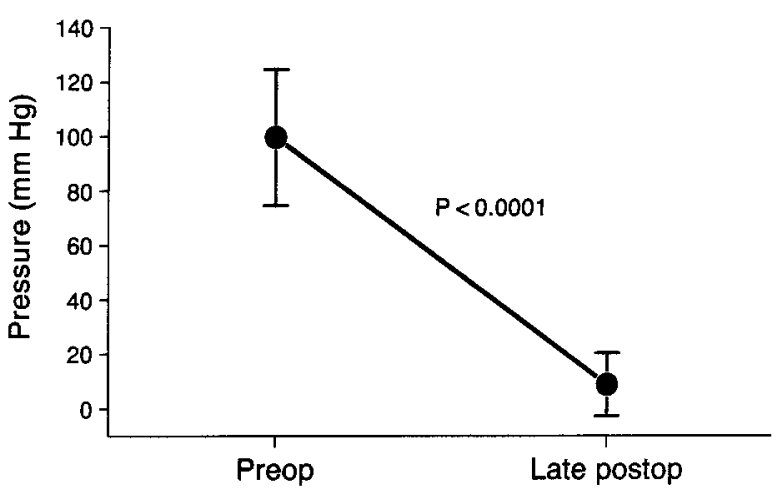

Fig. 5. Diagram of mean LVOT gradients measured before operation (Preop) and at late follow-up (Late postop) by echocardiography.

with angina $(p<0.001)$, and $5(100 \%)$ of 5 with syncope had complete relief of these symptoms after operation.

In accordance with a philosophy of maintenace of medical therapy after operation, 14 patients are being treated with $\beta$-adrenergic antagonists alone, 5 patients with calcium channel antagonists alone, 2 patients with both, 3 patients with amiodarone in combination with either $\beta$-adrenergic antagonist or calcium channel antagonist, and 1 patient with a combination of disopyramide and a calcium channel antagonist. At most recent contact, all patients had normal sinus rhythm.

Late echocardiographic information was available in 24 patients a mean of $5.3 \pm 4.5$ years after LVM. Mitral valve insufficiency was present in 9 patients (mild in 8 , moderate in 1) and mild aortic insufficiency was present in 3 patients. The mean LVOT gradient was $14.2 \mathrm{~mm} \mathrm{Hg}$ and the median was 5.0 $\mathrm{mm} \mathrm{Hg}$. Excluding the youngest patient, who was 2 months old at operation and who was the only patient with class III symptoms at follow-up, the mean LVOT gradient was $8.9 \mathrm{~mm} \mathrm{Hg}$. These reductions in gradients (measured by echocardiography) compare favorably with the mean premyectomy gradient of $99.9 \mathrm{~mm} \mathrm{Hg}$ (measured by echocardiography or cardiac catheterization, or both $(p<$ 0.0001) (Fig. 5).

\section{Discussion}

The clinical course and prognosis of HOCM in children, as in adults, is highly variable because of a wide spectrum of anatomic and physiologic abnormalities. Maron and coworkers ${ }^{12}$ have suggested that in children and adolescents with HOCM the rate of progression of the disease may be more rapid 


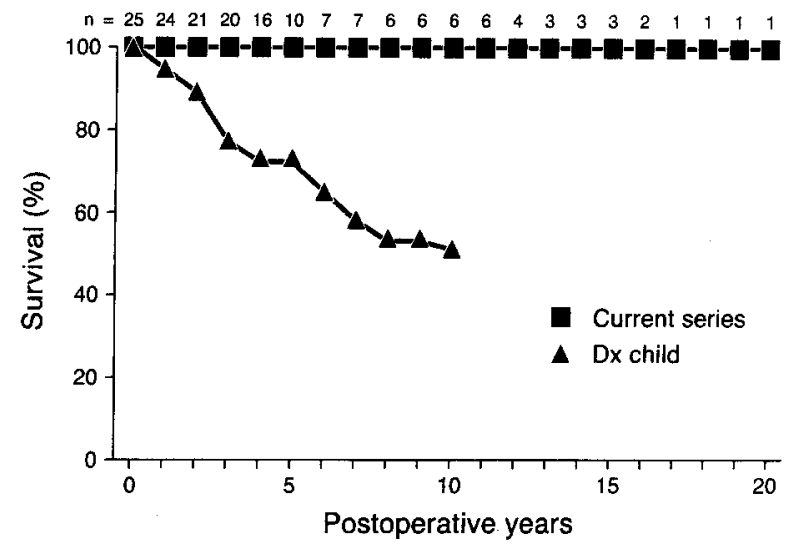

Fig. 6. Patient survival in current surgical series compared with survival in pediatric patients with HOCM treated nonsurgically ( $D x$ child) as reported by McKenna and associates. ${ }^{14}$

than in the adult HOCM population and that the factors responsible for body growth and development characteristics of childhood and adolescence may have an important role in inducing this progression.

Negative inotropic agents including $\beta$-adrenergic antagonists, calcium channel antagonists, and disopyramide have been used in an attempt to reduce the degree of LVOT obstruction in patients with HOCM. ${ }^{7,13}$ Although these agents have improved symptoms in many patients, there is little evidence that they improve patient survival. ${ }^{13,14}$ Treatment of children and young adults with HOCM is complicated by several factors including noncompliance with long-term medical therapy, intolerance to the medications (especially $\beta$-adrenergic antagonists), continued participation in strenuous physical activities, and an increased risk of sudden death compared to older patients. ${ }^{14-16}$

Sudden cardiac death is an unpredictable event in HOCM and is the most common mode of death in patients with this disease. ${ }^{14,17}$ The reported annual mortality rate for adult patients with unoperated HOCM is $3 \%$ to $4 \%$ per year, ${ }^{14,17}$ but for children with HOCM the mortality rate is $4 \%$ to $6 \%$ per year. ${ }^{14}$ Shah and coworkers ${ }^{17}$ found no correlation between severity of symptoms and the occurrence of sudden death. McKenna ${ }^{14}$ and Fananapazir ${ }^{18}$ and their colleagues have identified a history of syncope, a family history of sudden death, and the diagnosis of HOCM in childhood as prognostic markers for sudden death. Congestive heart failure in infants with HOCM is a particularly unfavorable prognostic sign; 9 of 11 such infants died in the first year of life in the report by Maron and coworkers, ${ }^{16}$ and the only patient in the present series who was not in class I or II at follow-up was the patient who was born with severe heart failure. Maron and colleagues $^{15}$ found that 23 of 26 patients without symptoms who died suddenly were younger than age 25 years. The presence of obstruction may also be a factor in sudden death; in one review of 31 children with hypertrophic cardiomyopathy, sudden death occurred in $40 \%$ of patients with obstruction compared with only $8 \%$ of patients without obstruction. ${ }^{19}$

It is generally agreed today that transaortic septal myectomy is the most appropriate surgical treatment for patients with HOCM and severe symptoms unresponsive to medical therapy. ${ }^{3-7,20}$ Most surgical articles report results and long-term survival after myectomy in adults or in patients over a wide age spectrum and do not offer a separate analysis of results for pediatric subsets. Two previous publications from our institution addressed the outcome in children and young adults after operation for HOCM. Fiddler and associates ${ }^{21}$ reported the cases of 16 patients younger than age 20 years who underwent operation for HOCM. There were four deaths, one early and three late, over a 16-year follow-up giving an annual mortality rate of $1.6 \%$ per year. In the same report, 13 children had received no surgical therapy, and 7 died. Mohr and coworkers $^{22}$ evaluated the results in 47 young patients with ages ranging from 1 to 38 years who underwent operation for HOCM. There was no early mortality; two late deaths occurred during a follow-up that ranged up to 16 years for a linearized mortality rate of $0.3 \%$ per year. There has been no early or late mortality in the present review. These results compare favorably with the expected mortality rate for pediatric patients who do not undergo operation of $4 \%$ to $6 \%$ per year (Fig. 6). ${ }^{14}$

Other institutions have also reported late results after operation in children that are better than the expected mortality for patients who do not undergo operation. Stone and colleagues ${ }^{8}$ at the National Institutes of Health analyzed the outcome in 17 pediatric patients who underwent operation because of HOCM. There was 1 perioperative death and 5 late sudden deaths ranging from 3.8 to 21 years after operation; the annual mortality rate was $1.8 \%$ per year. Williams and Rebeyka ${ }^{23}$ reported results in 25 children with ages ranging from 2 months to 17 years 
who underwent myectomy because of HOCM; there was no early death and only 1 late death, which occurred at reoperation 9 years after initial myectomy, in a follow-up that ranged up to 20 years.

The patients in the present series were given some type of negative inotropic medication after operation, even though the LVOT gradient and the mitral insufficiency were satisfactorily relieved. This philosophy was based on our historical observation that late survival in young patients was lowest before the development of medical or surgical therapy and was better when surgical treatment, especially LVM, became available. Survival was best when LVM was combined with postoperative administration of $\beta$-adrenergic antagonists or calcium channel antagonists when these medications became available. The improved results with the combined surgical and medical therapy were thought to be a result in part of suppression of sudden late, presumably arrhythmic, deaths. ${ }^{4,8}$ However, there is a recent trend in our institution among some cardiologists to discontinue administration of all negative inotropes in adults after successful LVM. Clearly, more data are required, preferably from randomized trials, regarding the appropriateness and effectiveness of treatment with postoperative negative inotropes in preventing late arrhythmic deaths.

In most instances, mitral insufficiency associated with HOCM is reduced to a negligible degree when the LVOT obstruction is successfully abolished with septal myectomy. In the present series, mitral insufficiency was minimal or absent in all patients but one at late follow-up. The single exception was the youngest patient who was born with severe cardiac failure and was 2 months old at initial operation; in this case LVOT obstruction redeveloped and new obstruction in the right ventricular outflow tract occurred. In some patients with long-standing mitral insufficiency associated with HOCM, a deformity of the anterior leaflet of the mitral valve is produced. This can often be corrected with a plicating suture. ${ }^{9}$ Occasionally, mitral valve replacement is required when there are organic changes in the mitral valve that are not amenable to mitral valve repair. In general, we prefer to avoid mitral valve replacement whenever possible because of the potential problems of thrombosis, thromboembolism, durability, infection, and anticoagulation related to prosthetic mitral valves.

Transaortic septal myectomy may present special problems in young patients because of limited exposure through the aortic valve anulus. Special care must be taken to avoid injury to the delicate aortic valve cusps during retraction for exposure of the hypertrophied septum, a complication that may necessitate subsequent aortic valve replacement. ${ }^{8,22}$

Another special technical consideration in young patients relates to accurately judging the depth and length of the septal myectomy. Inadequate excision will leave residual LVOT obstruction and limit symptomatic improvement and patient survival. In our experience, adequate myectomy may be achieved in essentially all patients, but careful hemodynamic assessment after weaning from cardiopulmonary bypass is mandatory for optimal results. Reinstitution of bypass and resection of additional septal muscle should be considered if the residual peak systolic gradient exceeds $20 \mathrm{~mm} \mathrm{Hg}$. Too deep a resection will create a ventricular septal defect $^{3,6}$; this complication can readily be detected by intraoperative transesophageal echocardiography and then corrected at the same operation.

Complete heart block is another complication that can be produced by traction against the aortic anulus or ventricular septum or by placement of the initial septal incision too close to the membranous septum. Despite the anatomic constraints created by a small aortic root in pediatric patients, the prevalence of permanent complete heart block can be kept low (no instances in the 25 patients in the present series).

LVOT obstruction may recur in pediatric patients after initial successful myectomy, but the prevalence is unknown. In the present series, two children required repeat septal myectomy 3.2 and 12.4 years after the initial procedure, respectively. Both patients survived and currently remain free of LVOT obstruction. Williams and Rebeyka ${ }^{23}$ suggested that children appear to have an increased risk of reoperation for LVOT obstruction in that 3 of 25 patients reported in their review required repeat myectomy with a range from 10 months to 9 years after initial operation. They compared these results with those in 139 adults who underwent septal myectomy for HOCM, none of whom required repeat operation because of recurrent gradients. Stone and associates ${ }^{8}$ reported provokable LVOT gradients of 40 and $48 \mathrm{~mm} \mathrm{Hg}$ in two pediatric patients at follow-up after septal myectomy.

Recently, dual-chamber pacing has been proposed as an alternative to surgical treatment for relief of symptoms in patients with HOCM. ${ }^{24,25}$ An acute decrease in gradient may be produced by atrioventricular sequential pacing with appropri- 
ately timed PR intervals, and symptomatic improvement has been reported with short-term follow-up. The mechanism responsible for the decrease in LVOT gradient is believed to be the abnormal septal motion produced by early pacing at the right ventricular apex. Also, there may be a late reduction of gradient related to ventricular remodeling and dilation..$^{25}$ If effective in the long term, dual-chamber pacing would be appealing because it would avoid heart operation, it could be done by all cardiologists and surgeons with pacemaker expertise, and it would not require specialized referral centers. However, if dual-chamber pacing fails to provide an effective reduction of cardiac symptoms and LVOT gradient, operation is then required, and the patient has the added expense, inconvenience, and risk of another procedure.

Short-term studies at our institution have shown favorable results in some patients after dual-chamber pacing. ${ }^{26}$ However, hemodynamic deterioration has occurred in other patients in whom the LVOT gradient increased, the pulmonary artery pressure increased, and the cardiac output fell. In nearly all patients, diastolic function deteriorated and left atrial pressure increased; the long-term implications of these findings are unknown.

Although symptoms may be relieved in some patients after dual-chamber pacing, there are differences in the degree of reduction of the LVOT gradients compared with that obtained with surgical myectomy. In a previous report from our institution, myectomy produced an $87 \%$ decrease in mean gradient, ${ }^{3}$ much greater than the $56 \%$ decrease achieved by pacing, ${ }^{24,25}$ and the mean residual gradient was less with operation $(9 \mathrm{~mm} \mathrm{Hg}$ after LVM compared with $38 \mathrm{~mm} \mathrm{Hg}$ with pacing). In the present series, there was an immediate $89 \%$ reduction in intraoperative LVOT gradient to a mean residual gradient of $6.6 \mathrm{~mm} \mathrm{Hg}$ after myectomy. The relief of LVOT obstruction was maintained in most patients at late follow-up; the mean echocardiographic maximum instantaneous gradient $(14.2 \mathrm{~mm}$ $\mathrm{Hg}$ ) was reduced $86 \%$ from the mean preoperative gradient $(99.9 \mathrm{~mm} \mathrm{Hg})$. The relatively high residual LVOT gradient in patients treated with dual-chamber pacemakers may be important. A residual gradient of $15 \mathrm{~mm} \mathrm{Hg}$ or greater was found to be an independent predictor of mortality after surgical myectomy in a previous report from our institution. ${ }^{3}$

Mitral insufficiency contributes substantially to symptoms of dyspnea in many patients with HOCM; the present review and previous reports from our institution $^{3}$ and others ${ }^{20,23}$ demonstrate that septal myectomy alone provides significant reduction of mitral insufficiency in most patients. It is not known how effectively dual-chamber pacing reduces the severity of mitral insufficiency. In addition, there are no data available yet to show that dual-chamber pacing reduces the threat of sudden death or improves survival of patients with HOCM.

The comparative merits of septal myectomy versus dual-chamber pacing are now being discussed. ${ }^{27}$ As the experience with dual-chamber pacing increases and lengthens, results will be compared with those of the more than 20-year experience with septal myectomy. Prospective studies to evaluate the mechanism and efficacy of dual-chamber pacing and proposed randomized trials of pacemaker versus myectomy will help to determine the relative role of each in the treatment of children and adults with HOCM.

In summary, relief of LVOT obstruction was initially accomplished in all of the young patients in the present series, and $96 \%$ have had significant improvement in preoperative symptoms. There was no early or late mortality. We conclude that extended LVM is a safe and effective means of relieving cardiac symptoms, reducing LVOT gradient, and correcting mitral insufficiency in pediatric patients. Myectomy can be accomplished with a low incidence of recurrent LVOT obstruction, and it provides excellent late survivorship that compares favorably with the natural history of the disease.

We thank Duane M. Ilstrup, MS, for advice on the statistical analyses and Merrilee Rogotzke for excellent secretarial support in the preparation of this manuscript.

REFERENCES

1. Fananapazir L, Epstein ND. Genotype-phenotype correlations in hypertrophic cardiomyopathy: insights provided by comparisons of kindreds with distinct and identical $\beta$-myosin heavy chain gene mutations. Circulation 1994;89:22-32.

2. Watkins H, McKenna WJ, Thierfelder L, et al. Mutations in the genes for cardiac troponin $\mathrm{T}$ and alpha-tropomyosin in hypertrophic cardiomyopathy. N Engl J Med 1995;332:105864.

3. Mohr R, Schaff HV, Danielson GK, Puga FJ, Pluth JR, Tajik AJ. The outcome of surgical treatment of hypertrophic obstructive cardiomyopathy: experience over 15 years. J Thorac Cardiovasc Surg 1989;97:666-75.

4. Schulte HD, Bircks WH, Loesse B, Godehardt EAJ, Schwartzkopff B. Prognosis of patients with hypertrophic obstructive cardiomyopathy after transaortic myectomy: late results up to twenty-five years. J Thorac Cardiovasc Surg 1993;106:709-17.

5. Heric B, Lytle BW, Miller DP, Rosenkranz ER, Lever HM, 
Cosgrove DM. Surgical management of hypertrophic obstructive cardiomyopathy: early and late results. J Thorac Cardiovasc Surg 1995;110:195-208.

6. Robbins RC, Stinson EB. Long-term results of left ventricular myotomy and myectomy for obstructive hypertrophic cardiomyopathy. J Thorac Cardiovasc Surg 1996;111:586-94.

7. Wigle ED, Rakowski H, Kimball BP, Williams WB. Hypertrophic cardiomyopathy: clinical spectrum and treatment. Circulation 1995;92:1680-92.

8. Stone CD, McIntosh CL, Hennein HA, Maron BJ, Clark RE. Operative treatment of pediatric obstructive hypertrophic cardiomyopathy: a 26-year experience. Ann Thorac Surg 1993;56:1308-14.

9. McIntosh CL, Maron BJ, Cannon RO III, Klues HG. Initial results of combined anterior mitral leaflet plication and ventricular septal myotomy-myectomy for relief of left ventricular outflow tract obstruction in patients with hypertrophic cardiomyopathy. Circulation 1992;86(Suppl):II60-7.

10. Reis RL, Bolton MR, King JF, Pugh DM, Dunn MI, Mason DT. Anterior-superior displacement of papillary muscles producing obstruction and mitral regurgitation in idiopathic hypertrophic subaortic stenosis: operative relief by posteriorsuperior realignment of papillary muscles following ventricular septal myectomy. Circulation 1974;49/50(Suppl):II181-8.

11. Schoendube FA, Klues HG, Reith S, Flachskampf FA, Hanrath P, Messmer BJ. Long-term clinical and echocardiographic follow-up after surgical correction of hypertrophic obstructive cardiomyopathy with extended myectomy and reconstruction of the subvalvular mitral apparatus. Circulation 1995;92(Suppl):II122-7.

12. Maron BJ, Spirito P, Wesley Y, Arce J. Development and progression of left ventricular hypertrophy in children with hypertrophic cardiomyopathy. N Engl J Med 1986;315:610-4.

13. Kober G, Hopf R, Biamino G, et al. Long-term treatment of hypertrophic cardiomyopathy with verapamil or propanolol in matched pairs of patients: results of a multicenter study. Z Kardiol 1987;76:113-8.

14. McKenna WJ, Deanfield JE, Farugui A, England D, Oakley C, Goodwin J. Prognosis in hypertrophic cardiomyopathy: role of age and clinical, electrocardiographic, and hemodynamic features. Am J Cardiol 1981;47:532-8.

15. Maron BJ, Roberts WC, Edwards JE, McAllister HA, Foley DD, Epstein SE. Sudden death in patients with hypertrophic cardiomyopathy: characterization of 26 patients without functional limitations. Am J Cardiol 1978;41:803-10.

16. Maron BJ, Tajik AJ, Atwood GF, et al. Hypertrophic cardiomyopathy in infants: clinical features and natural history. Circulation 1982;65:7-17.

17. Shah PM, Adelman AG, Wigle ED, et al. The natural (and unnatural) history of hypertrophic obstructive cardiomyopathy: a multicenter study. Circ Res 1974;35(suppl 2):179-95.

18. Fananapazir L, Chang AC, Epstein SE, McAreavey D. Prognostic determinants in hypertrophic cardiomyopathy: prospective evaluation of a therapeutic strategy based on clinical, Holter, hemodynamic, and electrophysiological findings. Circulation 1992;86:730-40.

19. Maron BJ, Henry WL, Clark CE, Redwood DR, Roberts WC, Epstein SE. Asymmetric septal hypertrophy in childhood. Circulation 1976;53:9-19.

20. McIntosh CL, Maron BJ. Current operative treatment of obstructive hypertrophic cardiomyopathy. Circulation 1988; 78:487-95.
21. Fiddler GI, Tajik AJ, Weidman WH, McGoon DC, Ritter DG, Giuliani ER. Idiopathic hypertrophic subaortic stenosis in the young. Am J Cardiol 1978;42:793-9.

22. Mohr R, Schaff HV, Puga FJ, Danielson GK. Results of operation for hypertrophic obstructive cardiomyopathy in children and adults less than 40 years of age. Circulation 1989;80(Suppl):I191-6.

23. Williams W, Rebeyka I. Surgical intervention and support for cardiomyopathies of childhood. Prog Pediatr Cardiol 1992;1: 61-71.

24. Fananapazir L, Cannon RO III, Tripodi D, Panza JA. Impact of dual-chamber permanent pacing in patients with obstructive hypertrophic cardiomyopathy with symptoms refractory to verapamil and $\beta$-adrenergic blocker therapy. Circulation 1992;85:2149-61.

25. Jeanrenaud X, Goy J-J, Kappenberger L. Effects of dualchamber pacing in hypertrophic obstructive cardiomyopathy. Lancet 1992;339:1318-23.

26. Nishimura RA, Trusty JM, Hayes DL. Dual-chamber pacing for hypertrophic obstructive cardiomyopathy: a randomized double blind crossover trial. J Am Coll Cardiol. In press.

27. Nishimura RA, Danielson GK. Dual chamber pacing for hypertrophic obstructive cardiomyopathy: has its time come? [Editorial]. Br Heart J 1993;70:301-3.

\section{Discussion}

Dr. Michel N. Ilbawi (Oak Brook, Ill.). I congratulate the authors for these excellent results in children with this difficult problem. This important work brings to focus several points that are extremely important concerning the surgical management of obstructive cardiomyopathy in pediatric patients.

The first point relates to the timing of operation. In the past, the initial treatment of patients with this lesion was primarily medical in the early stages. Operation was reserved until symptoms occurred or there was a severe onset of left ventricular hypertension. Encouraged by the early results, such as reported by the authors, we have operated on these patients earlier in the course of the disease. We attempted to relieve the pressure gradient before significant myocardial fibrosis or damage occurred. We are hoping that with this early intervention we can improve on the long-term outcome of this difficult problem. Would the authors please comment on their present philosophy concerning timing of operation? Do they still wait until the development of severe left ventricular hypertension or onset of symptoms before they recommend operation, or have they liberalized their indications for operation?

Another important point is the extent of resection. We believe that aggressive muscle resection is needed to achieve adequate and lasting relief of obstruction. We thin the septum almost to the level of the intact endocardium on the right side. In a few patients we have used patch enlargement of the LVOT, the so-called modified Konno procedure, to deal with severe obstruction. Do the authors have objective guidelines of how deep the muscle resection should be?

The third point concerns mitral valve operation and abnormality. I notice that the authors elected to plicate the valve in only 2 of 17 patients with moderate or severe 
regurgitation. What are the authors' recommendations concerning when and what to do for the mitral valve?

The fourth point is that patients with obstructive cardiomyopathy that present in early life are a different subset of patients. They are categorized by severe obstruction and have different short- and long-term outcomes. Did the authors analyze their results with this subset of patients?

Finally, this is an intriguing paper in the sense that the data imply that operation has improved survival in this group of patients. Do the authors maintain that survival has improved in their experience despite the absence of exercise testing or dobutamine stress echocardiographic evaluation?

Dr. Theodoro. In regard to our present philosophy on the timing of operation, I believe with the favorable results operation has now been shown to achieve that patients will be referred for operation earlier in the clinical course than they were in the beginning of this series. Nevertheless, the principal reason for offering operation at our institution is still persistence of symptoms associated with significant outflow tract gradients after adequate medical therapy. However, a few patients without symptoms are accepted for operation if they have fixed resting gradients in excess of 80 to $100 \mathrm{~mm} \mathrm{Hg}$.

The depth of septal excision depends on the thickness of the basal septum. Before the availability of intraoperative transesophageal echocardiography, septal thickness was assessed by bimanual palpation through the opened aorta. Currently we can obtain exact measurement of septal thickness with intraoperative transesophageal echocardiography. It is our philosophy that resection of approximately two thirds of the thickness of the septal bulge is required for adequate relief of outflow tract obstruction.

We have not needed to use the modified Konno procedure for primary operation in patients with HOCM, but it is an effective technique for relieving recurrent or more complex forms of LVOT obstruction. In one patient in whom recurrent obstruction and unexplained severe aortic insufficiency developed 12 years after initial myectomy, reresection and a Konno procedure were done with the use of an aortic homograft. That patient is currently completely free of symptoms with a competent aortic valve 5 years after the second operation.

With regard to anterior leaflet plication, this is done if there is redundancy or distortion of the anterior leaflet that appears to contribute to LVOT obstruction. This is more apt to occur in the adult population with longstanding HOCM. In the absence of organic changes in the mitral valve, an adequate septal resection has relieved the mitral valve insufficiency in the vast majority of our patients. The mitral valve is evaluated again by transesophageal echocardiography after myectomy. Any residual mitral insufficiency is treated by standard repair techniques.

Clearly the infant subset is a difficult group of patients that has a poorer short- and long-term outcome. Dr. Maron from the National Institutes of Health has reported the cases of 11 patients with congestive heart failure in infancy caused by HOCM, nine of whom died within the first year of life. We had one infant patient in this subset, who was born with congestive heart failure.
Recurrent LVOT and also right ventricular outflow tract obstruction have developed in this patient 5 years after operation at age 2 months. She is the only patient in this series who was not in NYHA class I or II at follow-up.

With regard to the last question, we believe that the symptomatic improvement and especially the longevity of this series of surgical patients are clearly superior to those published on the natural history of children with HOCM who have symptoms and do not undergo operation.

Dr. Richard A. Jonas (Boston, Mass.). Would the authors be a little bit more specific on the indications for operation? They have suggested that there may be an indication to operate to enhance longevity, in other words, not just to relieve symptoms, which has been the traditional indication for operation. If we accept that, then what are their indications in terms of pressure gradient? For example, I note that some patients had a pressure gradient as low as $20 \mathrm{~mm} \mathrm{Hg}$ : are asymmetric septal hypertrophy, a $20 \mathrm{~mm} \mathrm{Hg}$ gradient, and no symptoms then sufficient indications to operate?

Dr. Theodoro. In the patients with symptoms, the lowest resting or inducible preoperative gradient was $50 \mathrm{~mm} \mathrm{Hg}$. (The gradient of $20 \mathrm{~mm} \mathrm{Hg}$ was measured intraoperatively during general anesthesia.) A gradient of $50 \mathrm{~mm} \mathrm{Hg}$, by itself, without associated symptoms, would not generally be considered an indication for operation. The results of this review and other surgical series clearly show that longevity is increased by operation in patients with symptoms with LVOT obstruction, but improved longevity has not been demonstrated for patients without symptoms or for patients with mild or absent gradients. The five patients without symptoms in our series had fixed resting gradients in excess of 80 to $100 \mathrm{~mm} \mathrm{Hg}$. The referring pediatric cardiologist urged operation, believing the prognosis for the patient would be as bad as for patients with valvular aortic stenosis and similar gradients. Although this reasoning seems logical, the numbers are small and statistical data are lacking.

Dr. William G. Williams (Toronto, Ontario, Canada). I would like to ask a question about the management of complex and potentially fatal arrhythmias. In our experience of 230 patients with this disease the results are almost identical to what you have reported today. When we stratify the results by age, the outlook for children younger than age 18 is not different from that of the overall series. The prevalence of reoperation is also identical to yours at $94 \%$ at 10 years.

Importantly, when we look at the stratification of the patients who have syncope, who comprised $20 \%$ of the current series, the long-term survival is identical in patients with syncope versus that in those without. As you pointed out, the natural history of the disease is for sudden death in many patients; these deaths are presumed caused by ventricular tachycardia. However, after myectomy, sudden death is uncommon in our experience. We have only had two sudden, unexpected deaths in more than 200 patients treated by operation.

In this series there was one AICD implantation done at the time of operation and two after operation, and I am interested in the follow-up in those patients. This becomes a problem in patients who have sudden death. Our attitude has been to take a wait-and-see approach, and so 
far that has been reasonably successful. In the three patients who did have AICD implantation, do the authors have follow-up on whether the AICD triggered on the basis of an arrhythmia? Have late ventricular arrhythmias been a problem in their experience?

Dr. Theodoro. Indications for implantation of an AICD are still evolving. In general, when ventricular tachycardia or ventricular fibrillation has been documented before operation, an AICD implantation is planned. Some cases of familial HOCM have a strong family history of sudden death at an early age; these patients are evaluated by preoperative electrophysiologic testing. If significant ventricular arrhythmias can be induced, an AICD implantation is planned. Some patients with serious postoperative ventricular arrhythmias also receive an AICD. With the advent of effective transvenous cardioverter defibrillators, our cardiologists have lowered their threshold for recommending an AICD considerably. In those patients who have received an AICD, the number of subsequent cardioversion/defibrillations has ranged from 0 to more than 22 .

Dr. George R. Daicoff (St. Petersburg, Fla.). I have just one comment and a question. The comment is we are getting a lot of resistance from our cardiologists saying that all the patients need is a pacemaker. This is an important landmark paper that should be used as a standard of comparison for any other therapy that is recommended.

The question I have is this: after 5 years of follow-up with echocardiograms and other diagnostic tests, what can the authors tell us about the free wall? This might give us a clue as to how much of the LVOT obstruction is important in creating secondary left ventricular hypertrophy versus how much of this is the basic underlying disease. Can the authors provide this information?

Dr. Theodoro. The issue of whether left ventricular free wall hypertrophy regresses after relief of LVOT obstruction is controversial. In our group's experience, we have no convincing evidence that there is any appreciable or consistent regression of left ventricular free wall hypertrophy on follow-up echocardiograms. It appears to us that the free wall hypertrophy seen before operation results entirely or predominantly from inappropriate hypertrophy caused by genetic abnormalities, and this does not change appreciably even when the left ventricular pressure is reduced to normal levels by successful myectomy. The data presented in the rare literature reports in which consistent regression is said to occur are not convincing to us. 